Hautarzt $2021 \cdot 72: 42$

https://doi.org/10.1007/s00105-020-04741-w Online publiziert: 28. Dezember 2020

(c) Springer Medizin Verlag GmbH, ein Teil von Springer Nature 2020

\section{Wie vermittele ich rasch und leicht verständlich komplexe Themen im ärztlichen Alltag? Wie gelingt es mir, dabei eine größere Gruppe an Personen mit unterschiedlichem Vorwissen, Ausbildungsstand und Lernverhalten anzusprechen und im besten Fall zu einem inhaltlichen Austausch anzuregen?}

Vielleicht kennen Sie das Konzept der One Minute Wonder (OMW) bereits aus der Pflege, aus der Rettungs- oder aus der Intensivmedizin: Hier sind die kurzen, innerhalb einer Minute zu lesenden und aufzunehmenden Fortbildungseinheiten bereits seit Jahren etabliert ([1, 2]; - Tab. 1). Durch ihre knappe, ansprechende Darstellung sind die OMW besonders geeignet, um sie z.B. auch in Arztzimmern an Druckern oder in Pausenräumen aufzuhängen und kurze Wartezeiten während der Arbeitsabläufe sinnvoll mit der Möglichkeit, sich fortzubilden, zu füllen.

Uns erscheint dieses visuell ausgerichtete Konzept mit kleinen Lerneinheiten auch für die Dermatologie sehr passend, sodass wir den Weg der Wunder in diesen Fachbereich bahnen möchten.

Unser Ziel ist es, jede Ausgabe der Zeitschrift Der Hautarzt mit einem One Minute Wonder anzureichern. Das erste OMW in diesem Heft befasst sich mit dem Thema „Wechselwirkungen in der dermatologischen Systemtherapie" [3]. Es fokussiert auf die Interaktionen bei der gleichzeitigen Einnahme von Anti-

Julie Kind' · Alexander Zink ${ }^{2}$

'Fachzeitschriften Medizin, Springer Medizin Verlag GmbH, Heidelberg, Deutschland

${ }^{2}$ Klinik und Poliklinik für Dermatologie und Allergologie am Biederstein, Klinikum rechts der Isar,

Technische Universität München, München, Deutschland

\title{
One Minute Wonder - ergänzende, effiziente Wissensvermittlung auf einen Blick
}

mykotika und Phenprocoumon. Dieses wichtige Thema der Übersichtsarbeit von Krause et al. [3] mit zahlreichen anschaulichen Online-Abbildungen ist eine so gute Vorlage, dass 2 weitere OMW zu oralen Retinoiden bzw. zur QT-Zeit-Verlängerung geplant sind.

Wir freuen uns, wenn wir auch Sie, die Autoren ${ }^{\star}$ innen und Leser ${ }^{\star}$ innen von Der Hautarzt, von dem Konzept der One Minute Wonder begeistern können. Die Zahl der interessanten, praxisnahen Beiträge in der Zeitschrift ist so groß, dass Sie

Tab. 1 Empfehlungen zum Erstellen eines One Minute Wonder (OMW). (Leicht mod. aus [2])

Fokussieren Sie die OMW auf Informationen, die innerhalb 1 min gelesen und aufgenommen werden können

Konzentrieren Sie sich auf einen Aspekt, z. B. Entlastungspunktion, EKG(Elektrokardiogramm)Ableitung, Sichtungskategorien, Teilaspekt einer Therapie

Nutzen Sie kurze Aufzählungen, keine langen Texte

Betonen Sie den wichtigsten Lernpunkt

Verwenden Sie große, aus der Ferne lesbare Schriftarten

Verwenden Sie keine Patientendaten

Stellen Sie sicher, dass die Grundsätze des Plagiats und des Urheberrechts beachtet werden

Bilder/Grafiken lockern das OMW auf und wecken Interesse beim Leser

QR-Codes zur Verlinkung auf weitere Informationen sind hilfreich auf vielseitige OMW-Themen gespannt sein dürfen.

Wir hoffen auf einen regen Austausch, ob im Arztzimmer, auf dem Weg zum Labor oder gern auch in dieser Zeitschrift.

\section{Korrespondenzadresse}

\section{Dr. med. Julie Kind}

Fachzeitschriften Medizin, Springer Medizin Verlag GmbH

Tiergartenstr. 17, 69121 Heidelberg,

Deutschland

julie.kind@springer.com

\section{PD Dr. Dr. med. Alexander Zink}

Klinik und Poliklinik für Dermatologie und Allergologie am Biederstein, Klinikum rechts der Isar, Technische Universität München Biedersteiner Str. 29, 80802 München,

Deutschland

alexander.zink@tum.de

Interessenkonflikt. J. Kind und A. Zink geben an dass kein Interessenkonflikt besteht.

\section{Literatur}

1. Rowlinson J (2014) The one minute wonder network. Clin Teach 11(5):332-335. https://doi. org/10.1111/tct.12160

2. Eppler F, Monninger M, Schempf B et al (2019) One Minute Wonder - Fachwissen to go. Notfal Rettungsmed 22:642-644. https://doi.org/10. 1007/s10049-019-00639-w

3. Krause K, Jahn K, Homey B (2021) Wechselwirkungen in der dermatologischen Systemtherapie. Hautarzt. https://doi.org/10.1007/s00105-02004726-9 